\title{
EL SÍ MISMO AMPLIADO Y DISEMINADO: INTRODUCCIÓN A LA TEORÍA DE LA VALORACIÓN
}

\author{
Hubert J.M. Hermans \\ University of Nijmegen \\ The Netherlands
}

\begin{abstract}
RESUMEN
En este trabajo se presenta una introducción a la teoria de la valoración con las formulaciones clásicas de William James sobre el sí mismo como punto de partida. En esta teoria dinámica y abierta, el si mismo es concebido como un proceso organizado de valoración, valoración que es una unidad de significado personal a los ojos de la persona que relata la historia de su vida. La teoría se describe, cronológicamente, en tres partes: (a) la investigación sobre los sueños, los mitos colectivos, y las aplicaciones prácticas en el ámbito de las quejas psicosomáticas, esbozan la primera fase del edificio de la teoria; (b) en la segunda fase, la teoria recibe un nuevo impulso a partir de la noción de Bakhtin de la novela polifónica, que desemboca en una concepción multivocal del sí mismo; (c) la reciente ampliación de la teoría es ilustrada por la influencia que los otros imaginarios tienen en el sistema de valoraciones personales, y por la discusión del caso de una cliente al borde de un trastorno de personalidad múltiple.
\end{abstract}

Palabras clave: si-mismo, teoria de la valoración, significados personales, autonarraciones, auto-valoración, auto-percepción, si-mismo dialogal.

\begin{abstract}
With William James' classic formulation on the self as a starting point, an introduction into valuation theory is presented. In this open and dynamic theory the self is conceived of as an organized process of valuation, a valuation being a personal unit of meaning in the eyes of the person telling his or her life story. The theory is chronologically described in three parts: (a) research on dreams, collective myths, and practical applications on psychosomatic complaints illustrate the first phase of theory building: (b) in the second phase the theory has received a new impults from Bakhtin's notion of the polyphonic novel leading to a multivoiced conception on the self; (c) the recent extension of the theory is illustrated by the influence of imaginal others in the personal valuation system and by a discussion of a case of a client who was on the border of a multiple personality disorder.
\end{abstract}

Key words: self, valuation theory, personal meanings, self-narratives, self-valuation, self- perception, dialogical self.

Correspondencia: Hubert J.M. Hermans, Department of Clinical Psychology and Personality, University of Nijmegen, P.O. Box 9104, 6500 HE Nijmegen, The Netherlands. E-mail: hhermans@psych.kun.nl. 
La búsqueda del insight del si mismo ha sido una preocupación omnipresente desde que la cultura griega emprendió su marcha triunfal sobre la civilización occidental. Cuando los ciudadanos de la Grecia Antigua buscaban consejo en el oráculo de Delfos eran recibidos con el saludo de "conócete a tí mismo". Una de las principales figuras del pensamiento griego, Sócrates, decia a sus discipulos que "la vida no examinada no merece la pena ser vivida", pues el auto-conocimiento configura el camino de la verdad, la virtud y la felicidad (Robins y John, en prensa).

No obstante, existe un gran riesgo de malinterpretación de la antigua y clásica sabiduría griega, particularmente cuando asumimos que el sí mismo es una entidad contenida-en-simisma, que tiene significado en si misma, y que puede ser comprendida como separada de su historia personal y del ambiente. Este si mismo separado puede observarse claramente en algunos de los trabajos de uno de los pensadores más influyentes de los siglos pasados, René Descartes. En su "Tercera Meditación" (1641/1984, p. 24) escribe: "Ahora cerraré los ojos, me cubriré los oídos, y me apartaré de todos mis sentidos. Eliminaré de mis pensamientos todas las imágenes de las cosas corporales...Contemplaré tales imágenes como vacuas, falsas, y sin valor. Conversaré conmigo mismo y me escudriñaré de la forma más intensa; y así intentaré conseguir, poco a poco, un conocimiento más intimo de mi mismo". Siguiendo estas líneas Descartes llegó a la certeza completa de su famoso Cogito, ergo sum ("Pienso, luego existo"). Sin embargo, como observó Levin (1988), el sí mismo cartesiano surgió como "un sí mismo de la razón completamente purgado del cuerpo y del sentimiento, un sí mismo sin sombras, un si mismo completamente transparente a si mismo, completamente auto-poseído, completamente seguro de si" (p. 15). Como sugiere la crítica de Levin, la visión de Descartes sobre el sí mismo implica no solamente una concepción dualista del cuerpo y el si mismo, sino también una separación entre el sí mismo y el ambiente $y$, en particular, entre el sí mismo y el otro.

Aunque Descartes tuvo una tremenda influencia, no sólo en el pensamiento filosófico, sino también en las ciencias sociales y natu- rales (véase Johnson, 1987), su influencia en la psicología del sí mismo está corregida, al menos de forma parcial, por William James. En concreto, el capitulo de James sobre el si mismo en sus "Principios de Psicologia" (1890) prepara el escenario para el estudio empírico de la psique humana durante el siglo $X X$. En la perspectiva de James es fundamental su distinción entre Yo y Mi, distinción que, de acuerdo con Rosenberg (1979), es clásica en la psicologia del sí mismo. Consideraremos en primer lugar esta distinción y demostraremos posteriormente cómo la teoria de valoración se construye sobre ella en estrecha correspondencia con los desarrollos más recientes en la psicología narrativa.

James (1890) consideró los términos Yo y Mi como los dos componentes principales del si mismo. El Yo, o el sí-mismo-como-conocedor, organiza e interpreta continuamente la experiencia de una manera puramente subjetiva. Tres rasgos caracterizan al Yo: continuidad, distintividad y volición. La continuidad del si- mismo-como-conocedor se manifiesta por un "sentido de identidad personal" y un "sentido de igualdad" a través del tiempo (p. 332). El sentimiento de distintividad, de tener una existencia separada de los otros, es también intrínseco del Yo. El sentido de volición personal se expresa por la apropiación y rechazo continuos de pensamientos, mediante los cuales el sí-mismo-como-conocedor funciona como procesador activo de la experiencia. Cada uno de estos rasgos (continuidad, distintividad y volición) implican la conciencia de la auto-reflexividad esencial para el si-mismocomo-conocedor (Damon y Hart, 1982).

Al definir el Mi, o si-mismo-como-conocido, James (1890) era consciente de que hay una transición gradual entre Mí y Mío. En una famosa afirmación, identificó el Mi como el sí mismo empírico que, en su más amplio sentido, es descrito como todo lo que la persona puede considerar como propio, "no sólo su cuerpo y sus atributos físicos, sino sus ropas y su casa, su esposa e hijos, sus antepasados y sus amigos, su reputación y trabajos, sus tierras y caballos, y el yate y la cuenta bancaria" (p. 291). Estos elementos primarios, o constituyentes, señalan para James un rasgo básico del si mismo: su ampliación. La incorporación de los constituyentes indica que el sí 
mismo no es una entidad bloqueada y cerrada al mundo, y con existencia en si misma sino, más bien, ampliada hacia aspectos especificos del ambiente (Rosenberg, 1979). Con esta visión sobre la ampliación del sí mismo, James trasciende las fronteras estrictas de un si mismo contenido-en-sí-mismo y "esencialista". Dado que el Mio (el cuerpo, otra persona) pertenece al si mismo (ampliado), el sí mismo, en su más amplio sentido, es parte del ambiente, y el ambiente es parte del si mismo.

A continuación, demostraré como la distinción Yo-Mi de James regresa en la teoria de la valoración como teoría dinámica, en la cual el proceso de la ampliación del sí mismo es de interés primordial. Expondré esta teoria en tres fases. Primero la resumiré tal y como como se desarrolló originalmente sobre la base de algunos principios fundamentales de los trabajos de James. Segundo, discutiré la noción de Bakhtin de la novela polifónica, un poderoso concepto que encierra la promesa de desarrollar la distinción jamesiana Yo-Mi hacia una concepción narrativa y 'dialogal' (dialogical) del si mismo. En tercer lugar, mostraré cómo esta perspectiva dialogal ha dado un nuevo impulso a la teoría de valoración de manera tal que dicha teoria ha ampliado su esfera y asi la otra persona no es simplemente externa al sí mismo (la noción cartesiana del si mismo versus el otro) sino que está incluida como parte intrinseca de un complejo proceso, dialogal y altamente dinámico del si mismo.

\section{LA TEORÍA DE LA VALORACIÓN: FORMULACIÓN ORIGINAL}

La teoría de valoración (Hermans, 1987a, b, 1988, 1989; Hermans y Hermans-Jansen, 1995) se inspira en pensadores filosófico-fenomenológicos como James (1890) y MerieauPonty (1945), y concibe el si mismo como un proceso organizado de valoración. El aspecto de "proceso" se refiere a la naturaleza histórica de la experiencia humana e implica un orientación espacio-temporal. El individuo vive en una situación presente y está, desde un punto especifico del tiempo y el espacio, orientado hacia el pasado y el futuro, y hacia el mundo que le rodea. El aspecto "organizacional" señala el hecho de que el individuo no sólo se orienta sucesivamente a diferentes partes de su situación espacio-temporal, sino que también reúne estas partes en una historia o auto-narrativa. En esta historia, las diversas experiencias son situadas como partes en un todo compuesto, en el cual a una experiencia se le concede un lugar más influyente que a otra. Al contar su historia, el individuo tiene la capacidad de la auto-reflexión, conectando entre si experiencias diferentes de momentos y periodos también diferentes, y organizándolas como partes de un sistema de significados personales.

El concepto central de "valoración" se refiere a cualquier unidad de significado en la cual se organizan los sucesos de una autonarrativa. Tiene una connotación positiva (agradable), negativa (desagradable) o ambivalente a los ojos del individuo. Las valoraciones personales, como construcciones subjetivas de experiencias personales, se refieren a un amplio abanico de fenómenos como: un dulce recuerdo, una actividad agradable, una buena charla con un amigo, una decepción en la relación con otro significativo, una fuente concreta de satisfacción en el trabajo, una minusvalia fisica, un ideal inalcanzable, etc.. Durante diferentes periodos de la propia vida, pueden emerger diferentes valoraciones porque el punto de referencia del individuo está cambiando constantemente. A través del acto de la narración auto-reflexiva, estas valoraciones son organizadas en un sistema de valoración. Este sistema puede ser visto como un compuesto de valoraciones constantes y cambiantes.

Se asume que cada valoración lleva consigo una connotación afectiva, esto es, cada valoración tiene cierto grado de involucración personal y refleja un conjunto personal de sentimientos (un perfil o afecto concreto). Cuando sabemos qué tipo de afectos son característicos de una valoración particular, sabemos algo acerca de la valoración en sí misma. Esto también implica que el significado afectivo de una valoración no puede ser separado de ella. ¿Cómo se originó esta concepción del sí mismo en el trabajo de James?

Recordemos la definición de James del si mismo como "...la suma total de todo lo que (el sujeto) puede llamar su ...". Tras esta afirmación, James inmediatamente añadió: "To- 
das estas cosas le producen las mismas emociones. Si crecen y prosperan, se siente triunfante; si menguan y desaparecen, se siente deprimido, no necesariamente en un mismo grado para cada cosa, pero, con mucho, de la misma manera para todas" (p. 291-292). En otras palabras, James concibe el si mismo como ampliado hacia una múltiple variedad de cosas, y reaccionando con el mismo conjunto de emociones. De forma similar, nosotros concebimos las valoraciones como la variedad fenomenológica de las preocupaciones relevantes para un individuo, asociadas con el mismo conjunto de estados afectivos ( $p$. ej. uno puede experimentar ansiedad y rabia en relación a su padre y a su superior).

\section{El Nivel de Organización Manifiesto versus el Latente}

La riqueza fenomenológica de las valoraciones personales que pueden variar no sólo entre individuos sino también en un mismo individuo en el tiempo y el espacio, representa el nivel manifiesto del si mismo. En el nivel latente existe, sin embargo, un número limitado de motivos básicos, que se reflejan en el componente afectivo del sistema de valoraciones. El estudio del componente afectivo puede, por lo tanto, revelar qué motivo particular está activo en una valoración concreta $y$ en el sistema como un todo.

Se han tomado en consideración dos motivos básicos al caracterizar el componente afectivo del sistema de valoración: el afán por la Auto-Importancia, o motivo S (auto-mantenimiento y auto-expansión), y el anhelo por el contacto y unión con el otro, o motivo $O$ (participación con otras personas y con el mundo circundante). Esta distinción, relativa a la dualidad básica de la experiencia humana, ha estado presente en los escritos de diversos autores: Baken (1966) veia el efecto y la comunión como principios dinámicos fundamentales; Angyal (1965) se apoyó en el concepto de autonomía (o auto-determinación) y homonomia (o auto-renuncia); Klages (1948) consideraba el "Bindung" (solidificación) y el "Lösung" (disolución) como los dos motivos humanos básicos; McAdams (1985) ha distinguido el poder y la intimidad como los motivos básicos en un contexto narrativo. Reciente- mente, han sido ampliamente debatidas e investigadas las dimensiones del individualismo-colectivismo y el idiocentrismo-alocentrismo, sugiriendo nuevamente el carácter básico de los motivos $\mathrm{S}$ y $\mathrm{O}$, (Triandis, Bontempo, Villareal, Asai y Lucca, 1988; Schwartz, 1990; Lau, 1992).

En la teoría de valoración se asume que los motivos básicos se reflejan en el componente afectivo de una valoración. Explicaremos esto con dos valoraciones diferentes:

"En casa frecuentemente obtengo lo que quiero siendo un poquito agresivo".

"Encuentro que es fantástico cuando pinto un buen cuadro"

Asumamos que los sentimientos de "Fortaleza" y "Orgullo" son indicadores generales del motivo de Auto-Importancia (motivo S). La presencia de estos sentimientos en ambas valoraciones es la prueba de que son expresiones del mismo motivo subyacente. Dicho de otro modo, las valoraciones pertenecen a aspectos bastante diferentes del si mismo (difieren en el nivel manifiesto), aunque se hallan enraizados en el mismo motivo básico (son similares en el nivel latente). En esta construcción teórica, el componente afectivo proporciona el puente entre motivación y valoración. El afecto asociado con una valoración puede ser considerado como una expresión de los motivos básicos del nivel latente.

Veamos ahora algunas valoraciones que representan el anhelo del contacto y unión con el otro (motivo $O$ ):

"Durante la tormenta en el Mar del Norte sentí un vínculo auténtico con mi hermano: me sentia perdido en los elementos, y aún asi me mantuve firme sin tener que luchar para ser fuerte"

"Cantar en un grupo: la forma en la que mejor expreso mis sentimientos"

Ambas valoraciones son del mismo cliente, aunque representan manifestaciones claramente diferentes del sí mismo, implican fuertes sentimientos de intimidad y amor. Si asumimos que estos sentimientos son indicadores del motivo $\mathrm{O}$, entonces las dos valoraciones se pueden considerar de nuevo como diferentes en el nivel manifiesto, pero no en el latente.

En estrecha correspondencia con los sentimientos $\mathrm{S}$ y $\mathrm{O}$, el bienestar, en la forma de la diferencia entre los sentimientos positivos 
y los negativos, juega un papel central en la teoria de valoración. La razón es que, en su camino hacia la realización de los motivos básicos, las personas se encuentren con obstáculos. Estamos orientados de manera intencionada hacia el mundo, pero los obstáculos nos impiden con frecuencia la consecución de nuestras metas, resultando en sentimientos negativos. Por otro lado, cuando conseguimos algo o tenemos éxito en la superación de los obstáculos y estorbos, somos recompensados con sentimientos positivos. Se supone que cada valoración está asociada con un patrón de sentimientos positivos y negativos, de manera que el énfasis en uno de los dos tipos de afecto puede proporcionar información acerca del alcance en el que nuestros motivos básicos son gratificados.

\section{Generalización e Idealización}

Hay dos conceptos, Generalización e Idealización, que representan de forma particular la naturaleza dinámica de la teoría de valoración y juegan un papel central en la metodología presentada en el siguiente articulo ${ }^{1}$. Cuanto más se generalice una valoración concreta como parte del sistema, más determinará el "Sentimiento General" de la persona en cuestión. Cuando uno pregunta a una persona cómo se siente en general, es altamente probable que ciertas experiencias concretas coloreen, más que otras, este sentimiento general. Por ejemplo, si la persona está viviendo un periodo de conflicto serio con su cónyuge, se presenta una buena oportunidad para que los sentimientos asociados con este conflicto determinen con mayor probabilidad el sentimiento general de la persona durante este periodo que, por ejemplo, la buena relación que esta persona mantiene con sus compañeros. En otras palabras, no todas las valoraciones son igualmente influyentes en el sistema. Cuanto más poder de generalización tiene una valoración, más influyente es el componente afectivo de dicha evaluación en colorear la forma en que la persona se siente en general, en cierto periodo de su vida.

De forma similar, las valoraciones pueden diferir en la ampliación de la idealización. La idea básica es que ciertas valoraciones se ajustan más con la manera en la que a un individuo le gustaría sentir. Las valoraciones que colorean el sentimiento ideal son, con frecuencia, diferentes de aquellas que influyen en el sentimiento general. Este es el caso concreto de cuando las personas atraviesan por un periodo en el que se enfrentan con problemas personales que afectan a sus "yoes" en grado significativo. Bajo tales circunstancias, el sentimiento ideal tiene típicamente una modalidad afectiva en contraste con la modalidad afectiva del sentimiento general.

La formulación original de la teoría de valoración, como ha sido presentada hasta aqui, puede verse como una elaboración de la distinción de James entre Yo y Mi. En términos de valoraciones cargadas afectivamente, la persona cuenta como un Yo una historia acerca del Mi. Dado que hay una transición gradual entre Mi y Mío, el si mismo se extiende hacia aspectos específicos del mundo circundante. Es decir, el si mismo es capaz de reflexionar como un Yo, acerca de aquellos aspectos del mundo que son sentidos como Mio. Para la teoría de valoración esto implica que la persona, como proceso espacio-temporal, se extiende hacia el mundo, y en la valoración, como proceso de construcción del significado, se incluyen aquellos aspectos del mundo que son sentidos como personalmente relevantes.

A continuación daré algunos ejemplos que ilustran la naturaleza dinámica de la teoría de valoración: el estudio narrativo de los sueños y los mitos, y el estudio clínico de las quejas psicosomáticas.

\section{Los Sueños como Valoraciones Pictóricas}

Uno de nuestros proyectos combinaba la teoria de valoración y el programa de investigación sobre los sueños de Foulkes (1978). En la concepción de Foulkes las realizaciones del soñar son ordinarias más que exóticas, diferenciadas más que unificadas y, sobre todo, expresables en palabras. Por lo tanto, Foulkes sugirió que podia ser provechoso centrarse en los muchos y fuertes paralelismos existentes entre los sueños y el pensamiento ordinario lingüisticamente guiado. Como Foulkes, que concebia los sueños como series de frases pictóricas, Hermans (1978b) analizó el sueño de un sujeto como una serie de valoraciones 
pictóricas, definiendo la valoración como una unidad de significado a los ojos de la persona. El sueño fue estudiado comparando las valoraciones pictóricas (p. ej.., "Subí por una alta escalera") con las valoraciones ordinarias de la vida diaria, que normalmente tienen un carácter más conceptual ( $p$. ej., "Pasé un test dificil"). Se suponía que la diferencia entre la valoración pictórica y la conceptual no estaba más que en variaciones en el nivel manifiesto, y que ambas estaban enraizadas en una base motivacional común del nivel latente. Los resultados mostraron, efectivamente, fuertes paralelismos afectivos entre símbolos oníricos concretos (valoraciones pictóricas) y las preocupaciones diarias de la vigilia (valoraciones conceptuales), sin que los sujetos fueran conscientes de estos paralelismos. Una persona, por ejemplo, describió vívidamente que estaba caminando tranquilamente por un muro de arena en una pequeña ciudad holandesa y que, de repente: "La arena del muro empezó a desplazarse; no podia impedirlo y quedé enterrada". Cuando esta frase fue incluida en su sistema de valoraciones, el perfil afectivo asociado a la misma arrojó una correlación muy alta con el perfil afectivo de una valoración conceptual referente a la relación con su marido: "John es siempre capaz de hacerme saber de manera exacta y desagradable qué espera que yo haga, y yo soy incapaz de hacerle saber que no puedo hacer eso o que no quiero hacerlo". Aparentemente, las dos valoraciones tenian el mismo significado desde la perspectiva del nivel latente.

Se encontró, además, que los simbolos oniricos (p. e., "la arena que la enterraba"), que estaban separados inicialmente, a los ojos del sujeto, de las valoraciones de la vigilia, eran, tras un periodo de auto-reflexión, incorporadas con significado como partes del sistema de valoraciones de la vigilia en una segunda auto-investigación, unos seis meses más tarde. En otras palabras, en el proceso de interrelacionar las valoraciones del sueño con las de la vigilia, las primeras recibian un significado personal concreto cuando eran situadas en el contexto de las segundas. En nuestro ejemplo, esta mujer hizo una segunda auto-investigación transcurridos doce meses después de la primera y describió, de esta forma metafórica, la relación con su marido: "Literal y figurativamente hablando, no tengo espacio suficiente con John". De hecho, ella usó la metáfora de ser enterrada en su sueño por una fuerza ampliada para expresar la falta de espacio que tenia en la relación con su marido. En términos generales, se encontró que los sueños proporcionan simbolos útiles para la expresión metafórica de los aspectos significativos de las situaciones vitales de los sujetos.

\section{Temas en las Historias Individuales y Colectivas: Conflicto y Amor}

En la tradición de la investigación sobre la motivación psicológica y la naturaleza "relatada" (storied) de la experiencia humana (Murray, 1938; Sarbin, 1986; Bruner, 1986), nuestro propio trabajo relaciona los temas básicos de las historias colectivas con los motivos psicológicos de las vidas de las personas. La asunción básica es que hay dos temas fundamentales en las narraciones colectivas, heroismo y amor, y que estos temas se reflejan en las motivaciones psicológicas de los individuos (auto-importancia y contacto y unión). En un estudio de las series de Goya, se observó que "La captura del bandido El Maragato" (Hermans, 1988) expresaba la polaridad de la victoria vs. la derrota, representando el tema de la auto-importancia. Se encontró que ese mismo tema estaba presente en las auto-narrativas de clientes individuales: la experiencia de victoria se expresaba en afirmaciones tales como "Mi estatus es aceptable pero no suficiente; quiero ir un poco más allá", o "Mis logros eran míos, y tenian valor (piano, deporte, estudio)". La experiencia de pérdida se expresaba en afirmaciones como, "Tengo la sensación de que John puede ser fuerte manteniéndome a mi débil", o "Me han arrollado la violencia y la agresividad".

Un procedimiento similar se siguió en una investigación del mito de Narciso (Hermans y Van Gilst, 1991). Se descubrió que la parte central del mito, Narciso mirándose en el agua, representaba la experiencia de amor insatisfecho (para distinguirse de la disfunción psicoanalitica del narcisismo), y puede ser considerada como la expresión de un anhelo existencial por relacionarse y unirse a otras personas y con uno mismo. Sobre la base de 
los perfiles afectivos derivados de la parte central del mito, exploramos si existian perfiles similares en las valoraciones individuales de los clientes. Se halló que el mismo tema de anhelo no satisfecho estaba presente también en afirmaciones concretas como "Pienso que es terrible que no pueda eliminar algo de la soledad de mi madre con mi alegria", o "Antes yo lo significaba todo para él, ahora él lo significa todo para mí; los papeles se han invertido". El estudio de Goya y el de Narciso sugieren que los temas básicos, expresados en relatos colectivos, están presentes también en las auto-narrativas de las personas individuales.

\section{El Significado Personal de los Problemas Psicosomáticos}

En el curso del tiempo, hemos estudiado una variedad de quejas psicosomáticas como partes de un sistema de valoraciones organizado. Desde una perspectiva clínica, un hallazgo típico ha sido que muchos clientes no tienen idea acerca de la significación psicológica o del origen de sus quejas. La respuesta a esta ignorancia ha sido desarrollar una estrategia que integre las quejas psicosomáticas como parte del sí mismo proponiendo al cliente incluirlas como una valoración aparte. En la mayoria de los casos los clientes han mencionado simplemente la existencia de estos problemas (p. ej., "mi dolor de cabeza" o incluso, más breve, "dolor de cabeza"), o los describen con una frase que no revela más que la frecuencia o grado de gravedad del problema (p. ej., "Tengo a menudo dolor en el corazón" o "Sufro de un dolor terrible en el estómago"). En una etapa posterior, la valoración que se refiere a la queja psicosomática es estudiada en el contexto de las valoraciones del otro para explorar relaciones significativas. En otras palabras, una inicialmente "valoración aislada" es integrada como parte del sistema mediante su conexión activa con las otras partes del mismo.

En uno de nuestros estudios (Hermans, 1995), Alice, una mujer de 54 años, llevó a cabo una auto-investigación durante un periodo de serias dificultades con su marido. Sus problemas matrimoniales empeoraron cuando éste, escritor, publicó una obra de teatro en la cual ella podia ser reconocida como uno de los personajes principales. Esto provocó un serio problema, por el que pidieron asistencia terapeútica. Ambos, Alice y su marido, decidieron llevar a cabo una auto-investigación como punto de partida de un número de sesiones en las cuales discutieron sus opuestos puntos de vista bajo la guía de un psicoterapeuta. Una de las valoraciones de ella hacia referencia a "un dolor en el cuello y la parte posterior de la cabeza”. Para estudiar la queja psicosomática en estrecha relación con las otras partes del sistema de valoraciones, correlacionamos el perfil afectivo asociado con la queja con los perfiles afectivos de todas las otras valoraciones del sistema. En tal análisis (llamado Análisis de Modalidad; véase el siguiente articulo ${ }^{2}$ ), se supone que las valoraciones que correlacionan más alto tienen una significación particular porque tienen perfiles afectivos similares a la queja y, por tanto, comparten un significado afectivo similar. Tomando en cuenta las valoraciones que correlacionan más alto, el significado personal de una queja psicosomática (o cualquier problema clínico) puede verse aumentado. En la primera auto-investigación de Alice, hubo dos valoraciones que mostraron correlaciones muy altas con el problema psicosomático. Una hacia referencia a su pasado: "Mi padre: evitaba los problemas y utilizaba a sus hijos en su lucha contra mi madre; él siempre se conformaba con la autoridad de ella y así reforzó nuestro desvalimiento como hijos". La segunda valoración hacia referencia a un problema en la relación con su marido: "En la obra de teatro de mi marido, no me reconozco tal como él me ha representado". Aunque las correlaciones no pueden ser interpretadas en términos de relaciones causales, indican que hay un significado común en el dolor, por un lado, y en la problemática relación con el padre y el marido, por el otro. En una segunda auto-investigación, realizada ocho meses tras la primera, se encontró nuevamente una alta correlación entre la misma queja psicosomática y la valoración modificada que hacia referencia a la relación con su marido: "Lamento que mi marido haya revelado nuestros problemas en público antes de que tuviéramos la oportunidad de discutir- 
los en privado". Como sugiere esta valoración, las objeciones de Alice estaban formuladas más directamente contra la obra de teatro. Fue hacia el final de la segunda investigación cuando Alice y el psicoterapeuta decidieron centrarse en el significado de la queja de modo más explícito. Esto se hizo centrándose en las valoraciones que correlacionaban más alto en la primera y la segunda investigación, e invitando a Alice a exponer con sus propias palabras el significado que el problema psicosomático y las valoraciones que correlacionaban más alto tenían en común. Esto dió lugar a la formulación siguiente:

"Mi dolor puede estar suprimiendo rabia o resistencia (el patrón básico de mi conducta). Expreso mi insatisfacción de una manera indirecta $y$, por tanto, sin fuerza. Esto significa que una resistencia más directa podria aliviar mi dolor."

Un rasgo peculiar de esta interpretación es que Alice no sólo interpreta la comunalidad afectiva existente entre su dolor y la relación con su marido, ella formula también la dirección en la cual cambiar su conducta. De esta manera está moviéndose gradualmente de la evaluación al cambio (para tratamientos más ampliados del significado personal de las quejas psicosomáticas y otros problemas clinicos, véase Hermans, 1995; y Hermans y Hermans-Jansen, 1995).

\section{EL SÍ MISMO DIALOGAL Y MULTIVOCAL: UNA PERSPECTIVA BAKHTINIANA}

La conceptualización del si mismo en términos narrativos puede ampliarse más haciendo referencia al trabajo del erudito literario ruso Mikhail Bakhtin (1973). El texto ruso original de la traducción de su libro "Problemas de la Poética de Dostoyevsky" data de 1929. La importancia de este trabajo para la psicologia ha sido discutido por Vasil'eva (1988), Florenskaya (1989), Wertsch (1991), y Hermans y Kempen (1993). En primer lugar mostraré que las ideas de Bakhtin representan una contribución significativa para la comprensión de la naturaleza dialogal del sí mismo, y después mostraré cómo este cuerpo de ideas influyó en el desarrollo posterior de la teoria de la valoración.

\section{La Novela Polifónica: La Naturaleza Multivocal de la Mente}

Bakhtin (1973) observó que Dostoyevsky, uno de los más brillantes innovadores de la forma literaria, creó una forma peculiar de pensamiento artístico: la novela polifónica. En las novelas de Dostoyevsky no hay un autor único, el mismo Dostoyevsky, sino varios autores o pensadores, Raskolnikov, Myshkin, Stavogin, Iván Karamazov, el Gran Inquisidor. Cada uno de estos héroes tiene su propia voz aireando su propio punto de vista, y cada héroe es autoritativo e independiente. Un héroe no es simplemente el objeto de la visión artística concluyente de Dostoyevsky, sino que da la impresión de ser el autor de su propia ideología. De acuerdo con Bakhtin, no hay una multitud de personajes dentro de un mundo objetivo unificado, iluminado por la visión individual de Dostoyevsky, sino una pluralidad de perspectivas y mundos: una polifonia de voces. Además, al igual que en una composición polifónica las diversas voces e instrumentos ocupan diferentes posiciones en el espacio y se acompañan y oponen los unos a los otros en forma de diálogo.

Para entender la naturaleza de la novela polifónica, deberíamos ser conscientes de la diferencia existente entre relaciones lógicas y dialogales. Bakhtin ofrece los siguientes ejemplos (véase también Vasil'eva, 1988). Considérense dos frases completamente idénticas: "la vida es buena" $y$, de nuevo, "la vida es buena". En los términos de la lógica aristotélica estas dos frases están conectadas por una relación de identidad; son, de hecho una misma afirmación. Sin embargo, desde una perspectiva dialogal pueden ser vistas como dos observaciones secuenciales que se siguen en el tiempo y que provienen de dos personas en comunicación, separadas en el espacio y que, en este caso, mantienen una relación de acuerdo. Las dos frases son idénticas desde un punto de vista lógico, pero son diferentes en tanto que declaraciones: la primera es una afirmación, la segunda es una confirmación. De manera similar podemos comparar las frases "la vida es buena" y "la vida no es buena". En un sentido lógico una es la negación de la otra. Sin embargo, cuando las dos frases son tomadas como las declaraciones de dos hablantes diferentes, puede verse que existe una 
relación dialogal de desacuerdo. En la visión que Bakhtin tiene del mundo, las relaciones de acuerdo y desacuerdo son, al igual que la pregunta y la respuesta, formas básicas dialogales.

La novela polifónica representa a cada personaje como intrínsecamente relacionado con otros personajes reales o imaginarios. Al transformar el pensamiento "interno" de un personaje particular en una declaración, se producen de forma espontánea relaciones dialogales entre esa declaración y la de los otros imaginarios. La novela de Dostoyevsky "El doble" puede servir como ejemplo. En esta obra, el segundo héroe (el doble) fue presentado como la personificación de la voz interior del primer héroe (Golyadkin). Al externalizar la voz interior del primero en un oponente separado espacialmente, puede desarrollarse un diálogo completamente nuevo entre las dos partes independientes. En este diálogo cada personaje puede contar, como autor independiente, una historia acerca de sí mismo. En esta construcción narrativa, Dostoyevsky presupone una pluralidad de consciencias e, igualmente, una pluralidad de mundos que no son ni idénticos ni están unificados, sino que son bastante heterogéneos e incluso opuestos. De acuerdo con este mecanismo, retrata personajes que conversan con el diablo (Iván y el Diablo), con sus alter egos (Iván y Smerdyakov), e incluso con caricaturas de ellos mismos (Raskolnikov y Svidrigailov).

\section{La Presencia de Figuras Imaginarias}

La relación con figuras imaginarias juega un papel más significativo en nuestras vidas que lo que las teorías psicológicas tradicionales nos hacen creer. En su libro, "Los invitados invisibles", Watkins (1986) argumenta que en la mayoría de las teorías psicológicas los fenómenos imaginarios se abordan la mayoría de las veces. Estas teorias dan una prioridad claramente ontológica a lo real y a la existencia de los otros reales, mientras que los otros imaginarios son vistos como derivados a partir de ellos y subordinados a ellos. No obstante, los diálogos imaginarios juegan un papel central en nuestras vidas cotidianas: coexisten junto a los diálogos reales que mantenemos con los otros reales $y$, entrelazados con interacciones reales, constituyen una parte esen- cial de nuestra construcción narrativa del mundo. Incluso cuando estamos aparentemente silenciosos nos encontramos a nosotros mismos comunicándonos con nuestros criticos, con nuestros padres, nuestras conciencias, nuestros dioses, nuestro reflejo en el espejo, con la fotografía de alguien que echamos de menos, con la figura de una película o de un sueño, con nuestros bebés, o con nuestras mascotas.

A pesar de su cualidad de invisibles, las figuras imaginarias se perciben de forma tipica como ocupando una posición espacial aparte. Esto se aplica no sólo a nuestra propia cultura (p. ej., la relación imaginaria con un padre o amigo fallecidos, con un amante ideal, con un sabio mentor), sino también a comunidades no occidentales. Watkins, basándose en el trabajo de Warneck (1909), ofrece el ejemplo de los Bataks de Sumatra que mantienen la creencia de que el espíritu, que determina el carácter y la fortuna del ser humano, es como una persona dentro de una persona. El espiritu no coincide con la personalidad del anfitrión y puede estar a menudo en conflicto con él o ella. Es experienciado con frecuencia como un ser especial que está dentro de la persona, con su propia voluntad y sus propios deseos. En las conciencias miticas un espiritu tutelar no es concebido como el "sujeto" de la vida interior de alguien, sino como algo objetivo, "que habita en el hombre, que está conectado espacialmente a él $y$, por lo tanto, puede también ser separado espacialmente de él..." (Cassirer, 1955, p.168. citado por Watkins, 1986).

Caughey (1984), un antropólogo social, ha considerado el papel de "los mundos sociales imaginarios" tanto en las culturas occidentales como en las no occidentales. Llevó a cabo investigación de campo en Fánakker, una pacifica isla en Micronesia, y en las Colinas de Margalla en Pakistán, y comparó estas culturas con la cultura norteamericana. Su conclusión fue que las interacciones imaginarias no están, de ningún modo, restringidas a las culturas no occidentales. De acuerdo con su estimación, el mundo social "real" de la mayoría de los norteamericanos incluye entre 200 y 300 personas (p. ej. familia, amigos, conocidos, colegas). Aún más, una multitud de otros imaginarios pueden existir en el mundo social 
de una persona. Caughey los divide en tres grupos: (a) figuras de los medios de comunicación con los cuales el individuo se involucra en interacciones imaginarias; (b) figuras puramente imaginarias producidas en sueños y fantasias; (c) réplicas imaginarias de padres, amigos o amantes a las que se trata como si estuvieran realmente presentes. Caughey, como Watkins, argumenta que existen diálogos imaginarios junto con las interacciones imaginarias (p. ej., "Si mi amigo pudiera verme ahora...") y pueden o no tener un lazo directo con la realidad.

\section{Una Multiplicidad de Posiciones en el Sí Mismo}

Inspirándose en las consideraciones previas, Hermans, Kempen, y Van Loon (1992) conceptualizan el sí mismo en términos de una multiplicidad dinámica de posiciones del Yo relativamente autónomas en un paisaje imaginario. En su forma más concisa, esta concepción puede ser formulada como sigue.

El Yo tiene la posibilidad de moverse, como en el espacio, de una posición a otra de acuerdo con los cambios en la situación y en el tiempo. Fluctúa entre posiciones diferentes $e$ incluso opuestas, y tiene la capacidad de dotar a cada posición de voz, de manera que pueden establecerse relaciones entre las posiciones. Las voces funcionan como personajes en interacción dentro de una historia. Una vez un personaje es puesto en movimiento en una historia, adopta una vida propia, asumiendo asi cierta necesidad narrativa. Cada personaje tiene una historia que contar, desde su propia postura, sobre las experiencias vividas. En tanto que voces diferentes, estos personajes intercambian información acerca de sus respectivos Mi(s) y sus mundos, dando como resultado un complejo, y narrativamente estructurado, sí mismo (pp. 28-29).

En contraste al sí mismo separado cartesiano, el sí mismo dialogal parte de la asunción de que hay muchas posiciones del Yo que pueden ser ocupadas por la misma persona. El Yo de una posición, además, puede estar de acuerdo, en desacuerdo, comprender, malinterpretar, oponerse, contradecir, preguntar, e incluso ridiculizar al Yo de otra posición. Esta otra posición puede ser un otro real o un otro imaginario. El sí mismo 'encarnado' (embodied), en contraste con el si mismo separado, está siempre atado a una posición concreta del espacio y en el tiempo (tanto físicos como mentales). En términos de Merleau-Ponty (1945), no hay un "regard survolant". Es decir, al estar encarnada, la persona no es capaz de "volar libremente por encima" de su posición en el espacio y el tiempo, y la naturaleza encarnada de la existencia queda definida como la base de la vida humana.

El sí mismo es "social", no en el sentido de que un individuo contenido en si mismo entra en interacción social con otras personas, sino en el sentido de que otras personas ocupan posiciones en un si mismo multi-vocal. El sí mismo no va a ser visto como un receptáculo sino como, tomando prestado un término de Bruner (1990), diseminado. La asunción es que el Yo no está sólo "aquí" sino también "alli" y que, debido al poder de la imaginación, el Yo puede actuar "como si" él o ella fuera el otro. Esto no ha de ser equiparado a "adoptar el papel del otro" (Mead, 1934) en la medida en que esta expresión implica que el Yo adopta la perspectiva real del otro, fuera del si mismo. Más bien, yo construyo otra persona o ser como una posición que puedo ocupar, y una posición que crea una perspectiva alternativa del mundo y de mi mismo. Esta perspectiva puede ser congruente o no con la perspectiva real del otro real (lo cual puede ser comprobado entrando en conversación con el otro). Hay que añadir, sin embargo, que el otro puede ser, en gran medida, producto de mi imaginación, y ser, incluso, completamente imaginario (en los sueños o el arte).

El si mismo dialogal es también una reacción al ideal occidental del si mismo como una "estructura centralizada en equilibrio" (Sampson, 1985). Como estructura idealizada, el si mismo occidental intenta controlar el ambiente desde una posición centralizada, tratando de esta manera de encontrar un equilibrio entre el si mismo y el ambiente. El si mismo dialogal contrasta con la noción del si mismo como centro de control. Las diferentes posiciones del Yo representan diferentes puntos de anclaje, las cuales, dependiendo de la naturaleza de la interacción, pueden organizarse una a otra en un punto concreto en el tiempo. 
Como Watkins explica (1986), la figura imaginaria no es simplemente una proyección. Puede afirmarse igualmente que la persona experiencia al otro imaginario para crearse a si misma. Goethe, por ejemplo, dijo en una ocasión: "Las canciones me hacen, no yo a ellas". Jung (1961) tenia un compañero imaginario, Filemón, con el cual mantenia frecuentes conversaciones: "Filemón y otras figuras de mis fantasias me hicieron darme cuenta de que hay cosas en la psique que yo no produzco sino que se producen a si mismas y que tienen su propia vida (Jung, 1961, p. 183, citado por Watkins, 1986).

Una restricción cultural fundamental en nuestra visión psicológica tradicional del sí mismo es, precisamente, la fuerte centralización tan típica del ideal occidental, individualista y racionalista, del yo. Esta centralización impone serias limitaciones a las relaciones entre los diferentes personajes de la autonarrativa como campo de posibilidades. Si es cierto que la educación occidental ampara el ideal de un si mismo confinado, altamente centralizado (Sampson, 1985), entonces este ideal puede tener implicaciones de largo alcance para los personajes que son admitidos como partes integrantes de un sí mismo en desarrollo. Una fuerte tendencia a la centralización organizará el sí mismo en una forma tal que habrá una posición del Yo que podrá dominar a las demás reduciendo, por tanto, la posibilidad de diálogo que requiere, para su total desarrollo, un alto grado de apertura para el intercambio y la modificación de perspectivas.

El encogimiento, culturalmente basado, del si mismo y su centralización tienen consecuencias prácticas directas. Muchos psicoterapeutas están bastante familiarizados con los problemas de clientes que suprimen "subpersonalidades" concretas (Rowan, 1990) negándoles, por lo tanto, "una voz". Clínicos y psicoterapeutas se ven enfrentados a menudo con el problema de la intelectualización, por el que los clientes parecen ser incapaces de "dejar hablar a sus sentimientos" o de utilizar su imaginación de manera productiva. Aunque estos problemas se pueden atribuir en parte a la historia personal del sujeto (Spence, 1982), hay factores culturales básicos que limitan claramente el desarrollo completo de las relaciones dialogales. En otras palabras, el si mismo dialogal se puede concebir como una posibilidad humana fundamental, aunque cultural y psicológicamente restringida. (Para una discusión teórica más elaborada del sí mismo multivocal, véase Hermans y Kempen, 1993; para una revisión de sus manifestaciones empíricas en la literatura psicológica reciente, véase Hermans, 1996).

\section{Resumen de los Pasos Teóricos}

Las consideraciones anteriores pueden ser resumidas distinguiendo tres puntos en nuestro razonamiento teórico. En primer lugar, James (1890) dió un paso crucial al trascender el dualismo cartesiano entre el si mismo y el otro al señalar la transición gradual entre Mi y Mío. Así, por ejemplo, "mi amigo", "mi esposa" y "mis hijos" no están simplemente fuera del si mismo sino que, más bien, funcionan como ampliaciones de éste (véase también Mead ,1934, para un posterior desarrollo del sí mismo social de James). En segundo lugar, en las pasadas décadas, partidarios de una aproximación narrativa, como Bruner (1986), Sarbin (1986), y Gergen y Gergen (1988), han propuesto estudiar el si mismo como una historia que la persona construye acerca de si misma. Sarbin (1986) ha propuesto traducir la distinción Yo-Mí a un marco narrativo. Su tesis es que James, Mead, Freud, y otros, enfatizaron la distinción entre el Yo y el Mí y sus equivalentes en otros idiomas europeos precisamente dada la naturaleza narrativa del si mismo. El pronombre personal Yo se refiere al autor, y el Mi al actor o figura narrativa. El Yo como autor puede construir imaginativamente una historia en la cual el Mi es el protagonista. Tal construcción narrativa es posible porque el Yo como autor puede imaginar el futuro y reconstruir el pasado y describirse a si mismo como actor (Crites, 1986). Además, la construcción narrativa es un medio para la organización de episodios, acciones, y relatos de acciones (Sarbin, 1986, p. 9). En tercer lugar, la metáfora de la novela polifónica se expande sobre la concepción narrativa del Yo como autor y del Mi como actor observado. Mientras que en la versión de Sarbin de la auto-narrativa, se asume que un único autor cuenta una historia 
sobre si mismo como actor, la concepción del sí mismo como novela polifónica va un paso más allá: permite a un único y mismo individuo vivir en una multiplicidad de mundos en los que cada mundo tiene su propio autor que cuenta una historia de forma relativamente independiente de los autores de los otros mundos. Además, los diversos autores pueden entrar en conversación entre si. Es decir, el sí mismo conceptualizado como una novela polifónica integra las nociones de narrativa $y$ de diálogo imaginarios.

\section{RETORNO A LA TEORÍA DE LA VALORA- CIÓN: CONTRASTANDO Y COMPLEMEN- TANDO PERSPECTIVAS}

La teoría de valoración no es una teoría acabada, fija para siempre. Al contrario, está diseñada como una estructura abierta, en desarrollo, que permite al investigador $y$ al clínico investigar una diversidad de fenómenos psicológicos como un proceso de organización, desorganización, y reorganización del sistema de valoraciones. Tras la formulación inicial de la teoría, la noción del si mismo dialogal, discutido en las secciones previas, estimuló su posterior ampliación. El núcleo central de esta ampliación es triple. Primero, se supone que el sí mismo está compuesto por una multiplicidad de voces, cada una capaz de formular un sistema de valoraciones especifico. Es decir, diferentes voces pueden tener diferentes memorias sobre el pasado, diferentes preocupaciones acerca del presente $y$ diferentes metas y planes de futuro. Segundo, se supone que las diferentes voces y sus sistemas de valoraciones correspondientes se encuentran en el nivel manifiesto de funcionamiento del si mismo, y que todos estos sistemas están bajo la influencia de los mismos motivos básicos en el nivel latente. Tercero, las diferentes voces, al producir diferentes sistemas de valoraciones a partir de sus perspectivas especificas, pueden intercambiar información y asi influenciarse mútuamente de manera dialogal. Discutamos dos ejemplos que se han originado a partir de la versión ampliada de la teoria: la relación con figuras imaginarias, y el fenómeno del trastorno de personalidad múltiple.

\section{Figuras Imaginarias como Posiciones Opuestas}

En uno de nuestros ültimos proyectos de investigación (Hermans, Rijks, y Kempen, 1993) comparamos dos mujeres que habian mantenido una larga relación con una figura imaginaria, resultando en un penetrante impacto en sus vidas. Una de las sujetos, Kathy, era una mujer divorciada de 31 años y criada en una familia de clase baja. No habia finalizado el bachillerato, trabajaba como diseñadora gráfica, y no tenia historial psiquiátrico. Participó en el proyecto de auto-investigación porque estaba interesada en explorar el papel que su figura imaginaria jugaba en su vida. Ella describia esta figura como "una guía que me ayuda a encontrar mi camino en la vida", y dijo que estaba en contacto con tal figura desde sus primeros años de preescolar. Kathy fue invitada a formular, partiendo de preguntas abiertas acerca de su pasado, presente y futuro (véase el siguiente articulo) ${ }^{3}$, un conjunto de valoraciones desde la perspectiva de su Yo habitual. A continuación fue invitada a formular, partiendo de las mismas preguntas, un conjunto de valoraciones desde la perspectiva de su figura imaginaria. La idea presente tras esta investigación era que la guía tenia una historia propia que contar y podia hacerlo en los términos de un sistema de valoraciones específico. Se halló que los sistemas de valoraciones para las dos posiciones (Kathy y su guia) eran bastante diferentes, pero no sin relación. Por ejemplo, desde su posición habitual, Kathy dijo acerca de su pasado: "Cuando estaba sola, construi un mundo de fantasia donde me hice una persona muy fuerte." Desde la perspectiva de su guia, dijo: "Como niña, Kathy era callada y reservada: yo jugaba con ella en su propio mundo." Pero además, la guía dijo: "A veces tenia que proteger a Kathy de sus fantasias." Tal y como se sugiere en este ejemplo, la guia no sólo se preocupaba por Kathy, sino que la corregia cuando ésta iba demasiado lejos en sus fantasias. En este caso, la guía no es simplemente el producto de una mente disfuncional, sino al contrario, una restricción en la fantasia de Kathy y una fuerza productiva en su auto-organización.

El segundo sujeto, Liza, una mujer soltera de 32 años, fue criada en una familia de clase baja. Tras acabar sus estudios universitarios 
el año anterior, se encontraba desempleada. Padecia una depresión moderada pero crónica y, por tal razón, mantenía algunos encuentros con un psiquiatra. Participó en la autoinvestigación porque estaba interesada en llegar a conocer a su figura imaginaria, la cual describia como "un padre protector" que la amaba y que cuidaba de ella cuando tenia problemas. Dijo que fue consciente por primera vez de esta figura cuando tenia 8 años, estando sentada en una iglesia. Desde su posición habitual Liza decía, por ejemplo: "Nunca recibi protección de mi padre; con frecuencia yo tenia que protegerle a él." El padre imaginario parecia tener una función compensatoria para Liza, y decia: "Queria que ella sintiera que pertenecía a un lugar, y que era valiosa." Los dos sistemas de valoración (el de Liza y el del padre imaginario) eran muy opuestos desde el punto de vista afectivo. Liza formulaba valoraciones muy negativas (sentimientos más desagradables que agradables), mientras que las valoraciones provenientes del padre imaginario eran muy positivas, es decir, asociadas mucho más con sentimientos agradables que con desagradables. Esto sugiere que la figura imaginaria está compensando una carencia afectiva.

Seis meses después de las auto-investigaciones iniciales, tanto Kathy como Liza llevaron a cabo una segunda investigación, de manera que pudieran estudiarse los cambios. Los resultados de estas segundas investigaciones sugirieron que Kathy habia incrementado, durante el intervalo, la relación con su guia, y fortalecido la cualidad interactiva de la relación. Liza, sin embargo, no se habia beneficiado mucho de la relación con su padre imaginario, y las valoraciones desde su posición habitual no mostraron ninguna mejora. Estos resultados sugieren que pueden haber diferencias individuales considerables entre las perso-nas en la calidad de la relación con una figura imaginaria, y que para una esta relación puede tener una influencia más beneficiosa en su salud mental que para otra.

\section{Dividiendo el Sí Mismo: El Caso de la Hechicera \\ Las nociones de "personalidad múltiple" y "trastornos disociativos" (para revisiones véan- se, Kihistrom, Glisky, y Angiulo, 1994; Putnam,}

1993; Roberts y Donahue, 1994) son particularmente relevantes en nuestra discusión, dado que implican una seria reducción de la integración del sí mismo, que ha sido fragmentado en piezas. Como Watkins (1986) ha argumentado, la principal diferencia entre los diálogos imaginarios (normales) y la personalidad múltiple (anormal) es que en esta última hay un monólogo secuencial, más que un diálogo simultáneo. En los primeros hay una simultaneidad de posiciones entre las cuales el Yo se mueve hacia adelante y hacia atrás para que, pregunta y respuesta, acuerdo y desacuerdo, y un proceso activo de negociación entre las diversas posiciones sean posibles. En el caso de la disfunción también hay diversas posiciones (p. ej., la "Eva White" y la "Eva Black" del famoso caso de Thigpen y Cleckly, 1954) pero, sin embargo, es prácticamente imposible que se produzca una interacción dialogal entre estas posiciones. El cliente con una personalidad múltiple puede contar a menudo algo acerca de la persona de la otra posición, pero lo hace presentándola como un objeto (objectifying). Un ejemplo es la frivola Eva Black que decía acerca de la decente Eva White: "Cuando salgo y me emborracho, ella se levanta con la resaca" (Thigpen y Clecky, 1954, p. 141). Como el ejemplo sugiere, no hay una cooperación real ni una interacción entre las dos posiciones, de manera que la persona de una posición hace cosas que están completamente fuera del control de la persona de la otra posición.

Como parte de un estudio comprehensivo sobre las valoraciones disfuncionales (Hermans y Hermans-Jansen, 1995), presentamos una descripción detallada de una cliente, Mary, que estuvo, durante el periodo de tratamiento, muy próxima a padecer un trastorno de personalidad múltiple. Tenia malos recuerdos de su padre, que era alcohólico, y se sentia invadida por el asco y el pánico cuando veia a un hombre borracho. En su adolescencia participó en un episodio de drogas que recordaba con el mismo pánico y asco ya que sufrió un abuso sexual. Presentaba grandes dificultades en contar cómo fue obligada a practicar el sexo, a veces incluso bajo la amenaza de un arma. Como reacción a estas experiencias se "protegía" a si misma llevando siempre puesto un tampón. Además, se bañaba muy frecuente- 
mente para "limpiarse". Sus problemas se agudizaron cuando se casó con un hombre al que queria mucho pero, en marcado contraste con sus sentimientos hacia este hombre, habia momentos en los que sentia un profundo asco hacia él. A veces, de forma bastante repentina e inesperada, experimentaba una feroz agresividad hacia él, más allá de su control. Cuando su marido dormía sentía un apremio casi incontrolable de asesinarlo. Cuando él estaba enfermo, en cama, sentía odio y una total falta de compasión (como si viera a su padre durmiendo la borrachera, como se dió cuenta más tarde). Habia veces en las que se sentia como una hechicera, una experiencia extraña que la asustaba, especialmente porque había momentos en los que la hechicera tomaba posesión casi total de ella. Estaba mortalmente asustada y a veces se sentia literalmente estrangulada por un poder que era más fuerte que "ella misma".

Tras una amplia discusión de su caso, Els Hermans como psicoterapeuta y yo mismo como psicólogo de la personalidad, decidimos proponerle llevar a cabo una auto-investigación desde la perspectiva de dos posiciones: desde su posición habitual como "Mary", y desde la posición de "la hechicera". La razón que justificaba esta propuesta era que, dada la división existente entre las dos posiciones (Mary y la hechicera), se esperaba una mejora de su si mismo fragmentado si: (a) las dos posiciones se distinguian en cuanto a sus deseos, metas y sentimientos concretos y, (b) podia establecerse un proceso de intercambio dialogal entre las dos posiciones, de manera que la hechicera pudiera tener la oportunidad de decir lo que quisiera. De esta manera, Mary podria tener en cuenta las necesidades de la hechicera, sin perder el control de sus vehementes impulsos.

Como parte de nuestra estrategia invitamos no sólo a Mary a estimar el significado afectivo de sus propias valoraciones, sino que también se invitó a la hechicera a dar su vivencia afectiva de las valoraciones de Mary. Por ejemplo, Mary decia: "Quiero intentar ver qué es lo que me da mi madre: hay sólo una yo." Esta valoración fue experimentada por Mary de forma muy positiva. La hechicera, sin embargo, experimentaba la misma valoración de manera muy negativa y sentía una feroz agresividad hacia la madre. Por otra parte, la hechicera decia: "Con mis suaves cualidades felinas tengo a mano aspectos vulnerables de los cuales derivo mi poder en un momento posterior (alguien me dice cosas que puedo utilizar de modo que consigo lo que quiero.)" Para la hechicera esta valoración estaba asociada con una gran cantidad del afecto de auto-importancia y con una gran cantidad de sentimientos positivos. Sin embargo, en relación a la misma valoración, Mary sentía un grado bastante bajo del afecto de auto-importancia y una gran cantidad de sentimientos negativos. En otras palabras, Mary y la hechicera discrepaban fuertemente acerca del significado afectivo de tal valoración.

Tras esta auto-investigación, discutimos con Mary dos ideas basadas en nuestro análisis de su doble auto-investigación. En primer lugar, se le aconsejó moverse (p. ej., hacer deporte, dar paseos en bicicleta, o caminar) para ampliar su espacio imaginario y expresar la terrible energia de la hechicera. En segundo lugar, le propusimos llevar un diario en el cual podria escribir sus observaciones diarias, para agudizar asi su percepción. En una fase posterior, empezó a poner a prueba acciones nuevas. Ofrecemos aqui, expresado con sus propias palabras, un ejemplo del tipo de estrategia que desarrolló para afrontar a la hechicera:

"Hace unos días Fred estuvo enfermo, en cama, con 39 y medio de fiebre, e incluso le habian salido ampollas en los labios por la fiebre. Le preparé el desayuno y se lo llevé arriba. Cuando entré en la habitación y lo ví tumbado en la cama, lo aborreci y pensé: "¡No pienses que me voy a quedar en casa por tí!" (había planeado salir para visitar a unos amigos en Amsterdam y pasar la noche alli). De pie, delante de la cama, pensaba en esto (con malignidad creciente) y me dí cuenta de que la hechicera venía de nuevo. Le dejé a Fred la bandeja y sali de casa para dar un paseo. Durante el paseo senti que podia descargar una gran cantidad de la energía de la hechicera. A la vez tuve tiempo para reflexionar con calma sobre la situación tal y como era: "Él está enfermo, me necesita, y yo quiero cuidar de él." Decidí comprarle un periódico. Cuando llegué a casa le expliqué que pasaria la noche en casa y que iria a Amsterdam a la mañana siguiente. (Es decir, que no le dejé 
a él la decisión, sino que la tomé yo misma). Fred estuvo de acuerdo y a la mañana siguiente me fuí a visitar a mis amigos." (Hermans y Hermans-Jansen, 1995, p. 191).

Mary no sólo hizo una clara distinción perceptiva entre su posición y la de la hechicera, sino que también desarrolló una estrategia concreta para tratar con su oponente. Esta estrategia incluye que Mary no se desprende de, o suprime a, la hechicera, sino que más bien trata de estar tan alerta como le es posible a su aparición: cuando ésta se produce, Mary decide darse un respiro, pasea (moverse es importante para la hechicera) y luego toma una decisión más equilibrada.

Mary llevó a cabo una segunda investigación, transcurrido un año tras la primera, para evaluar los cambios. Durante el intervalo hubo un número limitado de sesiones en las cuales Mary discutía sus experiencias diarias con el psicoterapeuta. En la segunda investigación encontramos un aumento en el acuerdo existente entre las respuestas afectivas de Mary y las de la hechicera, tanto para las valoraciones de una como para las de la otra. Esto sugiere que se habia desarrollado una relación más dialogal entre las dos posiciones, y que Mary habia desarrollado un sistema de valoraciones más armonioso.

\section{CONCLUSIÓN}

La teoria de la valoración está diseñada como una teoría abierta y flexible para el estudio de un vasto conjunto de experiencias humanas. La teoria ha sido desarrollada recientemente mediante la toma en consideración de la multivocalidad del si mismo y la posibilidad de relaciones dialogales entre sus partes. La teoria proporciona no sólo un marco conceptual para distinguir diferentes posiciones del si mismo, también ofrece una estrategia concreta para la transición gradual entre la evaluación y el cambio. En lugar de omitir, suprimir, o separar posiciones incompatibles, se sigue un procedimiento en el cual se recogen las posiciones en un proceso dialogal. Como parte de esta estrategia, una posición incompatible no es simplemente "curada" o tratada como un síntoma indeseable, sino tomada seriamente como un compañero con el cual es posible coincidir "en términos ha- blados." El proceso dialogal, con la dominación y la lucha en él implicadas es, pues, un camino hacia la integración de posiciones incompatibles como parte de un sí mismo multivocal.

"Conócete a ti mismo" no era para Sócrates una empresa puramente "interna". Para mejorar su auto-conocimiento, salió a las calles y a los mercados y plazas a discutir con otras personas. Aún más, escuchaba a una "voz", su efecto moral, que le orientó durante gran parte de su vida. En cierto sentido, la concepción dialogal, multivocal, del si mismo está, a pesar de la distancia cronológica, más próxima a Sócrates que a Descartes.

\section{REFERENCIAS BIBLIOGRÁFICAS}

Angyal, A. (1965). Neurosis and treatment: A holistic theory. New York: Wiley.

Bakan, D. (1966). The duality of human existence. Chicago: Rnad-McNally.

Bakhtin, M. (1973). Problems of Dosoevsky's poetics (2nd ed.) Transl. R.W. Rotsel. U.S.A: Ardis. First published in 1929 under the title Problemy tvorchestva Dostoevskogo (Problems of Dostoevsky's Art).

Bruner, J.S. (1986). Actual minds, possible worlds. Cambridge, MA: Harvard University Press.

Bruner, J.S. (1990). Acts of meaning. Cambridge, MA: Harvard University Press.

Cassirer, E. (1955). The philosophy of symbolic forms. Vol.2: Mythical thought. New Haven, CT: Yale University Press.

Caughey, J.L. (1984). Imaginary social worlds: A cultural approach. Lincoln: University of Nebraska Press.

Crites, S. (1986). Storytime: Recollecting the past and projecting the future. In TH. R. Sarbin (Ed.). Narrative psychology: The storied nature of human conduct (pp. 152-173). New York: Praeger.

Damon, W. \& Hart, D. (1982). The development of self-understanding from infancy through adolescence. Child Development, 4, 841-864.

Descartes, R. (1984). Meditations on first philosophy. In J. Cottingham, R. Stoothoff, \& D. Murdoch (Eds. and Trans). The philosophical writings of Descartes (vol. 2, pp. 3-62). Cambridge, MA: Cambridge University Press. (Original work published 1641).

Florenskaya, T.A. (1989). Psychological problems of dialogue in light of the ideas of M. M. Bakhtin and A.A. Ukhtomskii. Soviet Psychology, 27, 2940. 
Foulkes, D. (1978). A grammar of dreams. Sussex, England: Harvester Press.

Gergen, K.J. \& Gergen, M.M. (1988). Narrative and the self as relationship. Advances in Experimental Social Psychology, 21, 17-56.

Hermans, H.J.M. (1987a). Self as organized system of valuations: toward a dialogue with the person. Journal of Counseling Psychology, 34, 10-19.

Hermans, H.J.M. (1987b). The dream in the process of valuation: A method of interpretation. Journal of Personality and Social Psychology, 53, 163175.

Hermans, H.J.M. (1988). On the integration of idiographic and nomothetic research method in the study of personal meaning. Journal of Personality, 56, 785-812.

Hermans, H.J.M. (1989). The meaning of life as an organized process. Psychotherapy, 26, 11-22.

Hermans, H.J.M. (1995). From assesment to change: The personal meaning of clinical problems in the context of the self-narrative. In R.A. Neimeyer \& M.J. Mahoney (Eds.) Constructivism in Psychotherapy (pp. 247-272). Washington, DC: American Psychological Association.

Hermans, H.J.M. (1996). Voicing the self: From information processing to dialogical interchange. Psychological Bulletin, 119, 31-50.

Hermans, H.J.M. and Hermans-Jansen, E. (1995). Self-narratives: The construction of meaning in psychotherapy. New York: Guilford Press.

Hermans, H.J.M. and Kempen, H.J.G. (1993). The dialogical self: Meaning as movement. San Diego: Academic Press.

Hermans, H.J.M., Kenpen, H.J.G, and Van Loon, R.J.P. (1992). The dialogical self: Beyond individualism and rationalism. Amercian Psychologist, 47, 23-33.

Hermans, H.J.M., Rijks, T.I., and Kempen, H.J.G. (1993). Imaginal dialogues in the self: Theory and method. Journal of Personality, 61, 207-236.

Hermans, H.J.M., and Van Gilst, W. (1991). Selfnarrative and collective mytg: An analysis of the Narcissus story. Canadian Journal of Behavioural Science, 23, 423-440.

James, W. (1890). The principles of psychology (Vol. 1). Macmillan.

Johnson, M. (1987). The body in the mind: The bodily basis of meaning, imagination, and reason. Chicago: The University of Chicago Press.

Jung, C.G. (1961). Memories, dreams, reflections. New York: Knopf.

Kihlstrom, J.F., Glisky, M.L. and Angiulo, M.J. (1994). Dissociative tendencies and dissociative disorders. Journal of Abnormal Psychology, 103, 117-124.

Klages, L. (1948). Charakterkunde (Characterology). Zürich, Switzerland: Hirzel.
Lau, S. (1992). Collectivism's individualism: value preference, personal control, and the desire for freedom among Chinese in Mainland China, Hong Kong, and Singapore. Personality and Individual Differences, 13, 361-366.

Levin, D.M. (1988). The opening of vision: Nihilism and the postmodern situation. New York: Routledge.

McAdams, D.P. (1985). Power, intimacy, and the life story: personological inquiries into identity. Chicago: Dorsey Pres (Reprinted by Guilford Press).

Mead, G.H. (1934). Mind, self, and society. Chicago: Univeristy of Chicago Press.

Merleau-Ponty, M. (1945). Phénoménologie de la perception (Phenomology of perception). Paris: Gallimard; Transl. in English by Colin Smith (1962). Pehnomelogy of perception. London: Routledge \& Kegan Paul.

Murray, H.A. (1938). Explorations in personality. New York: Oxford University Press.

Putnam, F.W. (1993). Dissociative disorders in children: Behavioral profiles and problems. Child Abuse \& Neglect, 17, 39-45.

Roberts, B.W. and Donahue, E.M. (1994). One personality, multiple selves: Integrating personality and social roles. Journal of Personality, 62, 199218.

Robins, R.W. and John, O.P. (in press). The quest for self-insight: Theory and research on accuracy and bias in self-perception. in R. Hogan, J. Johnson, and S. Briggs (Eds.). Handbook of personality psychology. New York: Academic Press.

Rosenberg, M. (1979). Conceiving the self. New York. Basic Books.

Rowan, J. (1990). Subpersonalities: the people inside us. London: Routledge.

Sampson, E.E. (1985). The decentralization of identity: toward a revised concept of personal and social order. American Psychologist, 11, 1203-1211.

Sarbin, Th. R. (1986). The narrative as a root methaphor for psychology. In Th. R. Sarbin (Ed.). Narrative psychology: the storied nature of human conduct (pp. 3-21). New York: Praeger.

Schwartz, S.H. (1990). Individualism-collectivism: critique and proposed refinements. Journal of Cross-Cultural Psychology, 21, 139-157.

Spence, D.P. (1982). Narrative truth and historical truth: Meaning and interpretation in psychoanalysis. New York: Norton.

Thigpen, C.H. and Cleckley, H. (1954). A case of multiple personality. Journal of Abnormal and Social Psychology, 49, 135-151.

Triandis, H.C., Bontempo, R., Villareal, M.J., Asai, $M$. and Lucca, N. (1988). Individualism and 
collectivism: Cross-cultural perspectives on selfgroup relationships. Journal of Personality and Social Psychology, 54, 323-338.

Vasil'eva, I.I. (1988). The importance of M. M. Bakhtin's idea of dialogue and dialogic relations for the psychology of communications. Soviet Psychology, 26, 17-31.
Warneck, M. (1909). Der Religion der Batak. (The religion of the Batak) Leipzig: T. Weicher.

Watkins, M. (1986). Invisibe guests: The development of imaginal dialogues. Hillsdale, $\mathrm{NJ}$ : Erlbaum.

Wertsch, J.V. (1991). Voices of the mind: A sociocultural approach to mediated action. London: Harvester Wheatsheaf.

\section{Nota del Autor}

Algunos lectores pueden preferir leer el segundo articulo sobre esta cuestión en primer lugar, dado que presenta el método concreto empleado en los ejemplos descritos en este primer artículo.

'Nota del editor: Decir que se publicará en un próximo número de la revista 